\title{
Patient friendly intensification of basal insulin
}

\author{
Sanjay Kalra \\ Bharti Hospital, Karnal, India \\ Correspondence to: Sanjay Kalra. Bharti Hospital, Karnal, India. Email: brideknl@gmail.com. \\ Provenance: This is an invited Editorial commissioned by the Section Editor Qi-Nan Wu (Chongqing University Cancer Hospital \& Chongqing \\ Cancer Institute \& Chongqing Cancer Hospital, Chongqing, China). \\ Comment on: Billings LK, Doshi A, Gouet D, et al. Efficacy and Safety of IDegLira Versus Basal-Bolus Insulin Therapy in Patients With Type 2 \\ Diabetes Uncontrolled on Metformin and Basal Insulin: The DUAL VII Randomized Clinical Trial. Diabetes Care 2018;41:1009-16.
}

Submitted Oct 15, 2018. Accepted for publication Oct 18, 2018.

doi: $10.21037 / \mathrm{atm} .2018 .10 .55$

View this article at: http://dx.doi.org/10.21037/atm.2018.10.55

\section{Background}

This is an exciting time in diabetology. Though the epidemic of type 2 diabetes epidemic is gradually converting to an endemic (1), there is unprecedented optimism in the diabetes clinic. Newer drug preparations, formulations, delivery devices, assisted by digital platforms and personfriendly approaches, are now available for management of this syndrome. These modern interventions can be used in multiple permutations and combinations $(2,3)$. Thanks to these medications, persons with type 2 diabetes now have a much wider, person-friendly, convenient choice of therapeutic options (4). These therapies allow safer and more convenient control, with a lower index of intrusion. They also act favorably on the associated metabolic morbidities, such as hypertension. dyslipidemia and obesity that often characterize diabetes.

In modern evidence based medicine, the onus of proof of efficacy of a particular drug lies upon the molecule in question. Randomized controlled trials, therefore, are necessary to prove that a specific drug can be used safely, in a particular group of patients.

\section{Study rationale}

Billings et al. set out to explore the efficacy and safety of insulin degludec liraglutide (IDegLira) co-formulation in persons with type 2 diabetes who are uncontrolled on metformin and basal insulin (5). Basal insulin is one of the second line drugs approved for use in persons with metformin inadequacy $(2,3)$. If even this proves inadequate, guidelines suggest intensifying to basal plus or basal bolus regimes. These regimes, however, are considered inconvenient by many patients, and are associated with unwanted weight gain and hypoglycemia. There is a need, therefore, to develop treatment algorithms which offer adequate glycemic control, with lesser number of injections, and with low risk of adverse events. The authors therefore chose to compare IDegLira with basal bolus insulin therapy.

\section{Study design}

The researchers described the results of a 32-week long phase $3 \mathrm{~b}$, multinational, open label trial, conducted in a two-arm, parallel, randomized manner. Participants were adults ( $\geq 18$ years) with type 2 diabetes, body mass index $\leq 40 \mathrm{~kg} / \mathrm{m}^{2}$, and poor control (HbA1c $7.0-10.0 \%$ ) in spite of stable daily doses of insulin glargine (20-50 U) and metformin ( $\geq 1.5 \mathrm{~g}$ or maximal tolerated dose) for $>90$ days prior to screening. Significant renal impairment (eGFR $<60 \mathrm{~mL} / \mathrm{min} / 1.73 \mathrm{~m}^{2}$ ) was taken as an exclusion criterion, as was use, or anticipated use, of medication known to affect weight or glucose metabolism. Use of glucose lowering drugs apart from glargine and metformin was ground for exclusion as well (5). This created a specific participant cohort which is representative of a large group of patients with basal insulin inadequacy. While one arm received basal bolus therapy, the other was randomized to treatment with IDegLira.

\section{Results}

Billings et al. demonstrated non-inferiority of glycemic control with IDegLira, as compared to basal bolus insulin 
in persons with type 2 diabetes, who were inadequately controlled on 20 to 50 units of basal insulin. Nausea, which is often a limiting factor for use of GLP1RA, was not seen to be a major concern in this trial. While nausea occurred in $11.1 \%$ of participants on IDegLira, versus $1.6 \%$ of basal bolus randomized participants, the reported nausea rate was $<3 \%$ at any given time point in both arms. No thyroid disease, pancreatitis, or major cardiovascular event was reported during the study (5).

\section{A major turning point}

This study marks a major turning point in our approach to diabetes care. The successful use of a single daily of fixed ratio combination injectable demonstrates that pharmaceutical technology has come of age. It is now possible to co-formulate two different drugs and administer them in a single patient-friendly device (6). Dose titration can be done using simple schemes which are easy to understand for the patient or his/her caregiver. Effective glycemic control can be obtained with much lower doses of insulin, thus allowing minimization of hypo glycemia and facilitation of weight loss. Improvements in systolic blood pressure and lipid profile, noted with IDegLira, add to the strength of this drug. Lesser number of injections, which can be administered flexibly, without regard to meal times or injection-meal gaps, enhance the attraction of this drug (7).

\section{Current consensus and recommendations}

The 2018 recommendations from the American Association of Clinical Endocrinologists (AACE)/American College of Endocrinologists (ACE) support optimization of both glycemia and weight, while minimizing hypoglycemia and weight gain. AACE clearly mentions that initial acquisition cost is not as important as the total cost of care, including the expenses incurred for screening detection and management of hypoglycemia (2). The need for combination therapy is highlighted, as is the importance of simple regimens, to optimize adherence. The importance of concomitant address of metabolic morbidities such as dyslipidemia and blood pressure is clarified as well.

The use of IDegLira adheres to all these principles of care. IDegLira is a simple, person friendly combination regimen which encourages adherence by its ease of use. It helps achieve composite targets of euglycemia and weight optimization, without hypoglycemia. IDegLira also exhibits multi-metabolic salutary effects as evidenced by it favorable impact on lipids and blood pressure. In all respects, therefore, the results of Billings et al. are concordant with the principles espoused by AACE.

The 2018 American Diabetes Association (ADA)/ European Association for Study of Diabetes (EASD) consensus approaches type 2 diabetes through the prism of vascular complications (3). The ADA/EASD rubric classifies persons with type 2 diabetes into those with, and without established atherosclerotic cardio vascular disease (ASCVD) and/or chronic kidney disease (CKD).

GLP1RA are mentioned as a first line treatment, after lifestyle modification and metformin. Authors clearly suggest that GLP1RA with proven cardiovascular benefits should be prescribed. Liraglutide is listed at second rank, after semaglutide, in hierarchy of choice. The use of liraglutide is supported over that of other GLP1RA including dulaglutide, exenatide and lixisenatide. Even in persons without ASCVD and/or CKD, GLP1RA figure as one of the therapeutic options after lifestyle modification and metformin. GLP1RA are strongly preferred when the aim of therapy is to minimize hypoglycemia and weight gain. If intensification with basal insulin is needed, ultra long acting basal analogues such as insulin degludec are preferred over insulin with shorter duration of action.

The study design followed by Billings et al. is based on basal insulin inadequacy, rather than GLP1RA inadequacy. It stands to reason, however, that the results support the choice of medication proposed by ADA/EASD.

ADA/EASD does not list GLP1RA as a therapeutic option in situation where cost containment is a prime concern. It must be noted though, that health economic studies analyze the total cost of care, including that of hypoglycemia and weight gain, rather than just the cost of glucose lowering drugs (8).

\section{Caveats}

We must keep in mind, however, that this study was performed in otherwise healthy community-dwelling adults, without renal impairment (eGFR $<60 \mathrm{~mL} / \mathrm{min} / 1.73 \mathrm{~m}^{2}$ ), who were not expected to initiate or change concomitant mediation known to affect weight or glucose metabolism. More work, therefore, needs to be done to explore the indications and relevance of IDegLira in a broader population of persons with diabetes. IDegLira must be compared in special populations, such as those with moderate renal impairment, and in persons taking concomitant obesogenic medication such as anti-psychotics, corticosteroids and 
immunosuppressants. It should also be assessed head-tohead with other commonly used insulin regimens, including basal plus and premixed formulations. Till then, the basal bolus regimen will continue to be a preferred option for indoor patients, those with eGFR $<60 \mathrm{~mL} / \mathrm{min} / 1.73 \mathrm{~m}^{2}$, and those on concomitant corticosteroid or immunosuppressant therapy, if intensification of basal insulin is needed

\section{Person-friendly option}

However, for the vast majority of persons into type 2 diabetes who need intensification of based insulin, IDegLira is as effective as basal bolus insulin. This efficacy is achieved with better safety (lower hypoglycemia) and tolerability (weight loss, as opposed to weight gain).

The current study provides robust evidence that patientfriendly, patient-centred, comprehensive metabolic and glycemic control is possible, with minimal adverse events, using a modern co-formulation like IDegLira. It adds strength of evidence to the experience of current recommendations, which signal a game changing approach in the management of diabetes.

\section{Acknowledgements}

None.

\section{Footnote}

Conflicts of Interest: The author has received speaker

Cite this article as: Kalra S. Patient friendly intensification of basal insulin. Ann Transl Med 2018;6(Suppl 1):S72. doi: 10.21037/ atm.2018.10.55 honoraria from Eli Lilly, Novo Nordisk and Sanofi.

\section{References}

1. Kalra S, Kumar A, Jarhyan P, et al. Endemic or epidemic? Measuring the endemicity index of diabetes. Indian J Endocrinol Metab 2015;19:5-7.

2. Garber AJ, Abrahamson MJ, Barzilay JI, et al. Consensus statement by the American association of clinical endocrinologists and American college of endocrinology on the comprehensive type 2 diabetes management algorithm - 2018 executive summary. Endocr Pract 2018;24:91-120.

3. Davies MJ, D'Alessio DA, Fradkin J, et al. Management of hyperglycaemia in type 2 diabetes, 2018. A consensus report by the American Diabetes Association (ADA) and the European Association for the Study of Diabetes (EASD). Diabetologia 2018. [Epub ahead of print].

4. Kalra S. Classification of non-insulin glucose lowering drugs. J Pak Med Assoc 2016;66:1497-8.

5. Billings LK, Doshi A, Gouet D, et al. Efficacy and Safety of IDegLira Versus Basal-Bolus Insulin Therapy in Patients With Type 2 Diabetes Uncontrolled on Metformin and Basal Insulin: The DUAL VII Randomized Clinical Trial. Diabetes Care 2018;41:1009-16.

6. Kalra S, Gupta Y. Injectable Coformulations in Diabetology. Diabetes Ther 2015;6:101-11.

7. Kalra S, Gupta Y, Unnikrishnan AG. Flexibility in insulin prescription. Indian J Endocrinol Metab 2016;20:408-11.

8. Jacobs E, Hoyer A, Brinks R, et al. Healthcare costs of Type 2 diabetes in Germany. Diabet Med 2017;34:855-61. 